\title{
Thermal Modeling of Snake Infrared Reception: Evidence for Limited Detection Range
}

\author{
B. S. Jones*, W. F. Lynn† And M. O. Stone $\$$ \$ \\ * Department of Biomedical Engineering, Wright State University, Dayton, OH 45435, U.S.A.; \\ $\dagger$ Veridian Engineering, 5200 Springfield Pike, Suite 200, Dayton, OH 45431, U.S.A. and \\ $\$$ Air Force Research Laboratory, Materials and Manufacturing Directorate, \\ Wright-Patterson Air Force Base, OH 45433-7702, U.S.A.
}

(Received on 28 October 2000, Accepted in revised form on 21 December 2000)

\begin{abstract}
For more than 40 years, information has circulated with regard to the sensitivity of infrared pit organs in both boid and crotaline snakes (pythons and pit vipers, respectively). The most often quoted sensitivity is $0.003^{\circ} \mathrm{C}$ and this value is based on the work of Bullock and co-workers (1956). Missing from previous work was a quantitative model of radiation transfer that would report sensitivity not in terms of degrees Celsius, but rather sensing distance. Since prey detection is often cited as the function of the infrared pit organ, quantification of this sensing distance seemed to be an important value that was missing from the literature. In this paper, we model the radiation transfer process from a $37^{\circ} \mathrm{C}$ object, i.e. warm-blooded prey, to an infrared pit organ. The model tries to answer a very basic question - at what distance does the thermal signature of a $37^{\circ} \mathrm{C}$ object blend into the background for a non-imaging biological infrared sensor? The output of the model, the sensing distance, is of particular interest in comparing biological infrared sensors to current semiconductor-based infrared (IR) detectors - largely because of inappropriate comparisons between the temperature sensitivity of IR snake reception and imaging IR cameras. The purpose of the presented work to make more appropriate comparisons, i.e. sensing distance. This sensing distance output indicates an extremely short detection distance $(<5 \mathrm{~cm})$ - contradictory to what is observed experimentally. This dichotomy raises further questions regarding how the biological system amplifies this weak signal.
\end{abstract}

(C) 2001 Academic Press

\section{Introduction}

Since the 1930s, scientists have been intrigued by the unusual facial pit organs found in the snake family Boidae and subfamily Crotalinae in family Viperidae (Noble \& Schmidt, 1937). This early work demonstrated that these organs were highly sensitive to thermal stimulation. Since then, the sensitivity of these organs has been an active area

$\S$ Author to whom correspondence should be addressed. E-mail: morley.stone@afrl.af.mil of research and debate. The sensitivity of crotaline (pit viper) infrared/thermal detection has been widely stated as $0.003^{\circ} \mathrm{C}$ and this value comes from the seminal work by Bullock and co-workers (Bullock \& Diecke, 1956). This threshold value was determined with water running over the pits - a convective mode rather than a radiant mechanism of heat transfer. This value has stirred debate as many researchers in the industrial infrared detector sector have questioned this sensitivity. Intrigued by the models put forth by de Cock Buning (1981, 1983a, b, 
1985), we sought to construct a radiation transfer model that would measure the radiant flux of a biological object as a function of distance.

The convective measurement of Bullock \& Diecke (1956) is certainly a proper method when measuring the sensitivity of a thermocouple, and temperature is an appropriate unit when measuring sensitivity. However, there are numerous underlying assumptions as follows. (1) The temperature of the sensor (a biological or physical mass) is changing due to IR radiation, thermal convection or thermal conductivity. (2) The temperature change produces a physical reaction (an electromotive potential or a neuron firing). (3) The physical reaction and temperature can be directly related to the source. In contrast, a semiconductor-based IR-photon detector does not change temperature due to IR radiation. As a result, a proper measure of the sensitivity for semiconductor-based IR-photon detectors is noise equivalent power or detectivity — radiometric quantities with no temperature equivalent. This debate is further confused when comparisons are made between pit organ sensitivity $\left(0.003^{\circ} \mathrm{C}\right)$ and the minimum temperature difference which an IR imaging system can distinguish, typically $0.05-0.10^{\circ} \mathrm{C}$.

We started from the assumption that the energy emitted from a $37^{\circ} \mathrm{C}$ object is a quantifiable entity and the transfer of that energy has been developed in disciplines outside the biological realm. Planck's distribution function is the standard form of expressing the spectral distribution of black-body radiation for an object at a particular temperature and this has been used for years in the infrared engineering community (Hudson, 1969). A $37^{\circ} \mathrm{C}$ object emits radiation maximally at $9.35 \mu \mathrm{m}$ or the far-infrared atmospheric transmission window.

The intent of the presented work was to develop a method for making system parameter comparisons, i.e. detection range or sensing distance. The difference between the method described herein and previously published work (de Cock Buning, 1983b) is the proper representation of atmospheric transmission and the radiation interactions between the stimulus, snake, and background within the field of view of the sensor. The source of this discrepancy is due to the use of the Stefan-Boltzmann law, which is an integra- tion of the Planck radiation law over all wavelengths and the field of view, representing all the radiation emitted by an object. Therefore, simply subtracting the results of the StefanBoltzmann formula for two objects of different temperature is only valid when describing the radiation exchange when the atmospheric absorption is negligible and the objects totally and exclusively interact with each other. Our use of configuration factors allows one to more accurately describe the energy transfer process that occurs with multiple emitting objects.

Snake infrared (IR) pit organs have been studied extensively for the function of prey detection (Theodoratus et al., 1997). We, along with other groups, have been involved in the study of infrared pit organ morphology in both boid and crotaline snakes (Campbell et al., 1999; Amemiya et al., 1996, 1995). Our results show the dramatic impact the thermal background has on detection properties of this biological system. In contrast, the output from our model is the determination of the distance between prey and detector that results in a $0.003^{\circ} \mathrm{C}$ change in temperature of the pit organ. We have chosen to express this threshold similar to Hartline (1974), and have divided the threshold temperature by the response time of $0.06 \mathrm{sec}$ to arrive at a rate of $0.05^{\circ} \mathrm{C} \mathrm{sec}^{-1}$.

Surprisingly, the output from our model indicates an extremely short detection range-less than $5 \mathrm{~cm}$. This result is in stark contrast to experiments performed with Agkistrodon species where detection ranges can be over an order of magnitude larger than what our model would suggest. This disconnect between our physical model and the actual biological response indicates that a unique biological signal amplification mechanism must be playing an integral role in the infrared pit organ's sensitivity. For example, it is not uncommon for biological systems to detect sub-nanomolar analyte concentrations via intracellular amplification mechanisms.

\section{Model and Results}

The results of our study are based on a modification of a model developed for describing the radiation transfer in a closed system, namely, an integrating sphere (Hisdal, 1965). In this model, radiation exchange between all of the surfaces is 


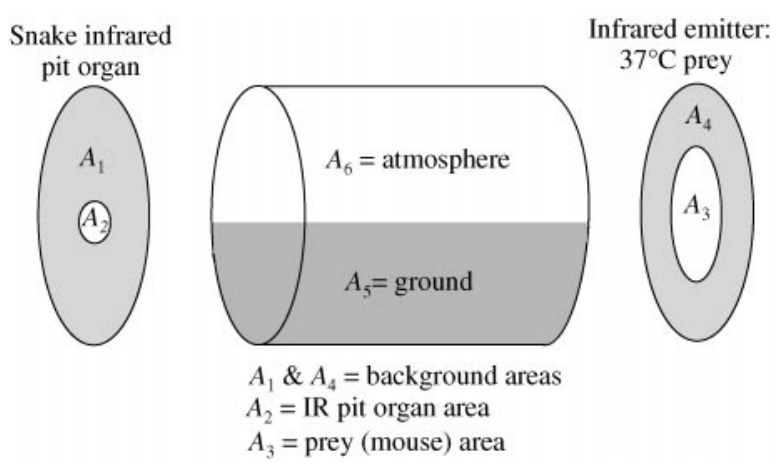

FIG. 1. Schematic drawing of the heat transfer model geometry. The emitter (prey) and detector (IR pit organ) are modeled using the configuration factors for a circular disk, and the energy transfer was modeled through a cylinder, half atmosphere and half ground. Exact dimensions are listed in the Modeling Results section.

reduced to knowing the reflectance of each surface and calculating configuration factors, i.e., the fraction of total radiant flux from a surface which irradiates another surface. The model described herein consists of six surfaces (see Fig. 1); therefore, 36 configuration factors are calculated. Areas 1, 4 and 5 are considered the ground or soil. Area 2 is the infrared pit organ membrane, area 3 is the prey, and area 6 is the sky or atmosphere. The appendix contains the configuration factors for the geometry illustrated in Fig. 1. The cylindrical geometry was chosen because of its applicability to burrows and similarity to soil undulations. Geometric configurations other than cylinders (cones with fixed apex angles or base diameters) were considered and analysed. In addition, various cylindrical aspect ratios were studied. These results are not presented because there is little difference in the final result $(<10 \%$ change in the sensing distance) and therefore did not affect our final conclusions.

The Hisdal method is a matrix representation of the radiant intensity, $L_{i}$, from each surface. In the original reference, the radiation exchange equations were simplified using Kirchhoffs Law for opaque surfaces, $r_{i}=1-\varepsilon_{i}$, and the reciprocity relation for Lambertian surfaces, $A_{i} F_{i j}=A_{j} F_{j i}$. The reader is referred to the original work for details of the mathematical rigor, since the following matrix equation is not intuitively obvious.

$\left(r_{i}(\lambda) F_{i j}-\mathbf{I}\right) L_{i}(\lambda)=-r_{i}(\lambda) E_{i}(\lambda), \quad i, j=1,6,(1 \mathrm{a})$ where $r_{i}$ is the reflectance of area $A_{i}, F_{i j}$ the configuration factor or fraction of radiation from area $A_{i}$ incident on area $A_{j}$, I the identity matrix, $L_{i}$ the radiant intensity of area $A_{i}$, and $E_{i}$ the irradiance of area $A_{i}$ from an external source.

The Hisdal method was developed for characterizing the radiometric errors of integrating spheres in the visible spectral region and includes variables for the external irradiation of any surface in the system. However, the method does not include terms which represent the black-body radiation from each surface. The active irradiation terms used by Hisdal are of the form $-r_{i} E_{i}$, where $r_{i}$ and $E_{i}$ are the reflectance and irradiance of the $i$-th element, respectively. These terms describe the radiation from active sources that are reflected by the surface into the system. If we consider thermally emitted radiation as an external contribution to the radiation equation(s), then substituting $-\varepsilon_{i} M_{i}$ for the external irradiation term properly describes the contribution of blackbody radiation by the surface into the system. Hence,

$$
\left(r_{i}(\lambda) F_{i j}-\mathbf{I}\right) L_{i}(\lambda)=-\varepsilon_{i}(\lambda) M_{i}(\lambda), \quad i, j=1,6,
$$

where $\varepsilon_{i}$ is the emissivity of area $A_{i}$, and $M_{i}$ the Planck distribution function (spectral radiant intensity) for area $A_{i}$.

The reflectance, emittance and Planck's distribution function are spectrally dependent on the spectral radiant intensity for a $37^{\circ} \mathrm{C}$ object (the temperature of warm-blooded prey) being maximal at $9.35 \mu \mathrm{m}$. However, it is unlikely that the snake is sensing a specific wavelength, but rather a broad range of wavelengths capable of being transmitted through the atmosphere. The maximum transmission through the atmosphere occurs in the 9-11 $\mu \mathrm{m}$ spectral region and falls to zero at 8 and $12.5 \mu \mathrm{m}$ due to absorption by atmospheric water vapor and carbon dioxide. Therefore, eqn (1b) must be integrated over this region. The reflectance values of the soil, prey, atmosphere, and snake infrared pit organ are inputs based on measured data or estimates and are nearly constant over this spectral region which simplifies the integration of eqn (1b). Integration of emissivity and Planck's distribution function are given by the following equation 
(Driscoll, 1978):

$$
-\varepsilon_{i} M_{i}=-\int_{\lambda_{a}}^{\lambda_{b}} \varepsilon_{i}(\lambda) M(\lambda, T) \mathrm{d} \lambda,
$$

where $M_{\lambda}(\lambda, T)$ is governed by Planck's radiation law:

$$
M_{\lambda}(\lambda, T)=c_{1} /\left[\lambda^{5}\left(\mathrm{e}^{c_{2} / \lambda T}-1\right)\right]
$$

and

$\lambda$ is the wavelength $(\mathrm{m}), c_{1}$ is equal to $3.7415 \times 10^{-16} \mathrm{~W} \mathrm{~m}^{-2}, c_{2}$ equal to $1.43879 \times$ $10^{-2} \mathrm{~m} \mathrm{~K}$, and $\mathrm{T}$ the temperature $(\mathrm{K})$.

Since reflectance, i.e. emittance, is not wavelength dependent for our model, the term $\varepsilon_{i}$ can be moved outside the integral in eqn (2) and the integral can be calculated over the interval from $\lambda_{1}$ to $\lambda_{2}$ (see Fig. 2), by the equation (Incropera \& DeWitt, 1996):

$$
\int_{0}^{\lambda_{2}} M_{\lambda} \mathrm{d} \lambda-\int_{0}^{\lambda_{1}} M_{\lambda} \mathrm{d} \lambda=\left(f_{\left(0 \rightarrow \lambda_{2}\right)}-f_{\left(0 \rightarrow \lambda_{1}\right)}\right) \sigma T^{4},
$$

where $f_{(0 \rightarrow \lambda)}$ is the fraction of total radiant intensity from a black body within a certain wavelength interval for a given temperature. From the interval $0-\lambda$, the fraction was determined by the ratio of the total area under the curve, as shown in Fig. 2.

The governing equation is

$$
f_{(0 \rightarrow \lambda)} \equiv \frac{\int_{0}^{\lambda} M_{\lambda} \mathrm{d} \lambda}{\int_{0}^{\infty} M_{\lambda} \mathrm{d} \lambda}=\frac{\int_{0}^{\lambda} M_{\lambda} \mathrm{d} \lambda}{\sigma T^{4}}
$$

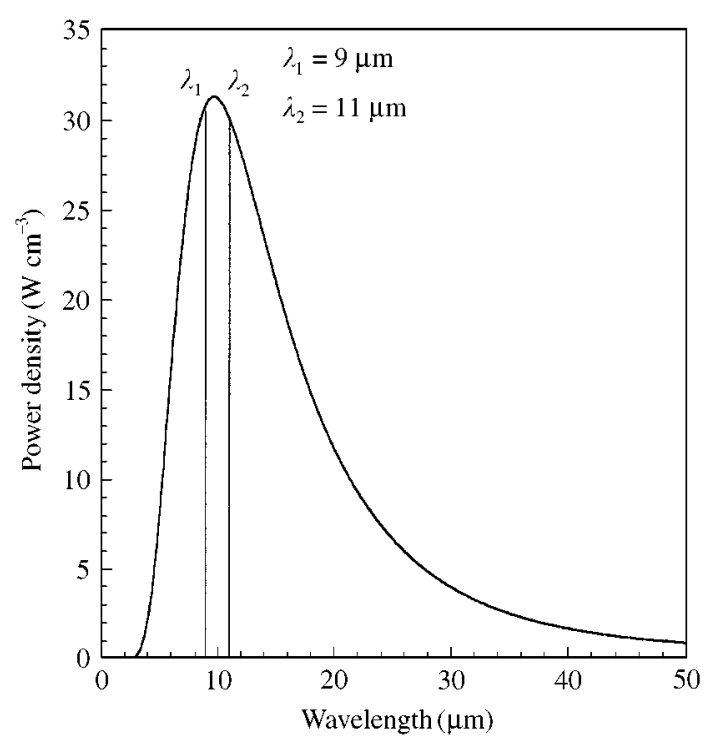

FIG. 2. Power density in $\mathrm{W} \mathrm{cm}^{-2}$, calculated using Planck's distribution function, plotted as a function of wavelength. The calculated curve is for a $310 \mathrm{~K}$ object and the interval used in this study, $9-11 \mu \mathrm{m}$, is marked on the

\begin{tabular}{|c|c|c|}
\hline Area & Temperature & Reflectance \\
\hline $\begin{array}{l}A_{1}=3.14 \mathrm{~m}^{2} \\
(\text { radius }=100 \mathrm{~cm})\end{array}$ & $T_{1}=290,295,300$ and $305 \mathrm{~K}$ & $\begin{aligned} r_{1}= & 1-\varepsilon(\text { soil })=1-0.9=0.1 \\
& (\text { Salisbury et al., 1994) }\end{aligned}$ \\
\hline $\begin{array}{l}A_{2}=3.14 \times 10^{-6} \mathrm{~m}^{2} \\
\text { (radius }=0.1 \mathrm{~cm})\end{array}$ & $\begin{array}{l}T_{2}=290,295,300 \text { and } 305 \mathrm{~K} \\
\text { (snake in equilibrium with the environment) }\end{array}$ & $\begin{array}{c}r_{2}=1-\varepsilon(\text { pit } / \text { skin })=1-0.971=0.029 \\
(\text { Togawa, 1989) }\end{array}$ \\
\hline $\begin{array}{l}A_{3}=2.83 \times 10^{-3} \mathrm{~m}^{2} \\
\text { (radius }=3 \mathrm{~cm} \text { ) }\end{array}$ & $T_{3}=310 \mathrm{~K}\left(37^{\circ} \mathrm{C}\right.$ object, warm-blooded prey $)$ & $\begin{array}{c}r_{3}=1-\varepsilon(\text { fur } / \text { mouse })=1-0.96=0.04 \\
\quad(\text { engineering estimate })\end{array}$ \\
\hline $\begin{array}{l}A_{4}=3.14 \mathrm{~m}^{2} \\
(\text { radius }=100 \mathrm{~cm})\end{array}$ & $T_{4}=290,295,300$ and $305 \mathrm{~K}$ & $\begin{array}{c}r_{4}=1-\varepsilon(\text { background soil })=1-1=0 \\
(\text { engineering estimate })\end{array}$ \\
\hline $\begin{array}{l}A_{5} \text {-depends on the } \\
\text { length of the tunnel }\end{array}$ & $T_{5}=290,295,300$ and $305 \mathrm{~K}$ & $\begin{aligned} r_{5}= & 1-\varepsilon(\text { soil })=1-0.9=0.1 \\
& (\text { Salisbury et al., } 1994)\end{aligned}$ \\
\hline $\begin{array}{l}A_{6} \text {-depends on the } \\
\text { length of the tunnel }\end{array}$ & $\begin{array}{l}T_{6}=263 \mathrm{~K} \text { (measured using AGEMA } \\
\text { Thermovision } 880 \text { IR camera }(8-12 \mu \mathrm{m})\end{array}$ & $\begin{array}{c}r_{6}=1-\varepsilon(\text { atmosphere })=1-1=0 \\
\text { (engineering estimate) }\end{array}$ \\
\hline
\end{tabular}
plot and spans the $\lambda_{\max }$ for a $310 \mathrm{~K}$ object.

TABLE 1

List of all values used in the model calculations for area, temperature, and reflectance 


\section{TABLE 2}

Black-body radiant intensity for the snake, prey, soil and atmosphere between the selected wavelength interval of 9-11 $\mu \mathrm{m}$. The total radiant intensity values calculated for all six objects in the heat transfer model

\begin{tabular}{lc}
\hline $\begin{array}{l}\text { Blackbody radiant intensity, } \\
9-11 \mu \mathrm{m}\end{array}$ & $\begin{array}{c}\text { Total radiant intensity } \\
L\left(\mathrm{~W} \mathrm{~m}^{-2} \text { ) }\right.\end{array}$ \\
\hline$M_{1}(\lambda, T)=61.73 \mathrm{~W} \mathrm{~m}^{-2}$ (soil) & $L_{1}=67.89$ \\
$M_{2}(\lambda, T)=61.73 \mathrm{~W} \mathrm{~m}^{-2}$ (snake) & $L_{2}=62.00$ \\
$M_{3}(\lambda, T)=72.35 \mathrm{~W} \mathrm{~m}^{-2}$ (prey) & $L_{3}=72.17$ \\
$M_{4}(\lambda, T)=61.73 \mathrm{~W} \mathrm{~m}^{-2}$ (soil) & $L_{4}=61.73$ \\
$M_{5}(\lambda, T)=61.73 \mathrm{~W} \mathrm{~m}^{-2}$ (soil) & $L_{5}=58.80$ \\
$M_{6}(\lambda, T)=31.31 \mathrm{~W} \mathrm{~m}^{-2}$ (atmosphere) & $L_{6}=31.31$ \\
\hline
\end{tabular}

where $\sigma$ is the Stefan-Boltzmann constant $\left(5.67 \times 10^{-8} \mathrm{~W} \mathrm{~m}^{-2} \mathrm{~K}^{-4}\right)$.

The areas, temperatures and reflectance values used as inputs to the model are listed in Table 1. These data were placed in the matrix [see eqn (1b)] and the radiant intensity $(L)$ was solved by Gaussian elimination using MATLAB $^{\circledR}$ software (Mathworks, Inc. 1984-1999). The fraction of radiant intensities calculated using eqn (4) for each area in the 9-11 $\mu \mathrm{m}$ spectral region and the results of the matrix calculation are presented in Table 2. A broader spectral range was also investigated $(8-12 \mu \mathrm{m})$, and no significant difference in the results was observed.

For a sensor organ to be of value for prey detection, the sensor must be able to distinguish the prey from the background. A useful sensor evaluation parameter is the distance at which the signal from the prey is equal to that from the background - the noise equivalent background distance. This distance can be determined by calculating the difference in radiant flux, $\Delta Q$, between the radiation reaching the sensor organ from the prey vs. the background as follows:

$$
\Delta Q=L_{3} A_{3} F_{32}-L_{6} A_{6} F_{62}-L_{5} A_{5} F_{52}-L_{4} A_{4} F_{42} .
$$

Figure 3 shows the change in radiant flux as a function of emitter to detector distance, when areas 1 and 4 are soil. This figure demonstrates how the radiant flux values decrease as the distance between emitter and detector is increased.

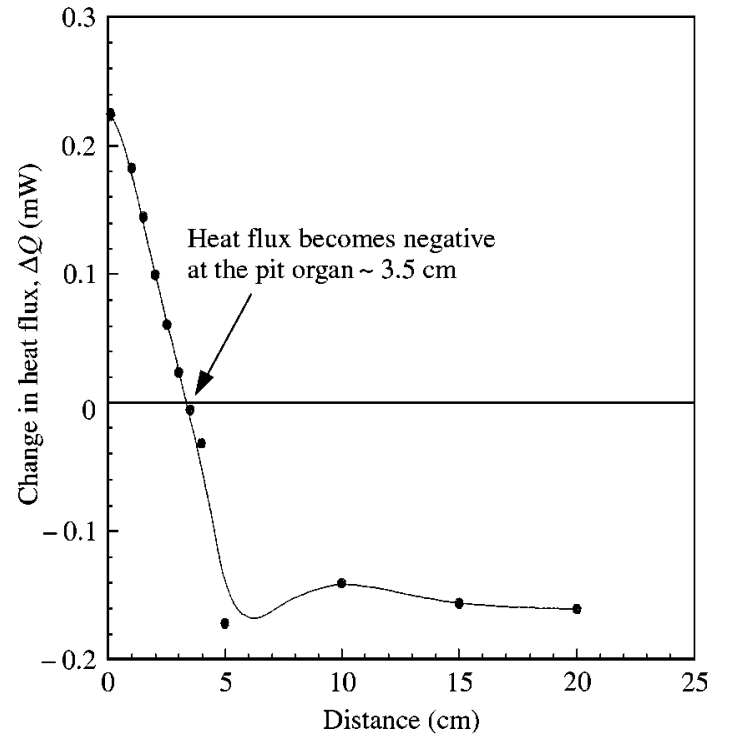

FIG. 3. The change in radiant flux, as measured at the snake's infrared organ, as a function of emitter distance. At negative $\Delta Q$ values, the emitter no longer has a signal greater than background.

This means that at around $3.5 \mathrm{~cm}$, the background radiation signal is greater than the prey, causing the change in radiant flux to become negative.

Under further analysis, the snake and soil temperatures were varied and the resulting effect was determined. Equation (6) was solved for various sensor-to-prey distances. The values of radiant flux for varying snake/soil temperatures are depicted in Figs 4 and 5. The results of these two figures show that as the emitter and detector distance is increased, the amount of radiation from the prey is reduced due to increased background radiation. In Fig. 4, for the case of areas 1 and 4 being soil, radiant flux became negative between 3 and $4 \mathrm{~cm}$. In Fig. 5, for the case of areas 1 and 4 being atmosphere, radiant flux became negative between 4 and $5 \mathrm{~cm}$. Therefore, these figures show that there was no significant difference in the radiant flux when varying the temperature and treating areas 1 and 4 as either soil or atmosphere.

The main question of interest was at what distance is there enough irradiated energy from a $37^{\circ} \mathrm{C}$ object incident on the pit membrane to cross a threshold of $0.05^{\circ} \mathrm{C} \mathrm{sec}^{-1}$ (Hartline, 1974; Bullock \& Diecke, 1956). To answer this 


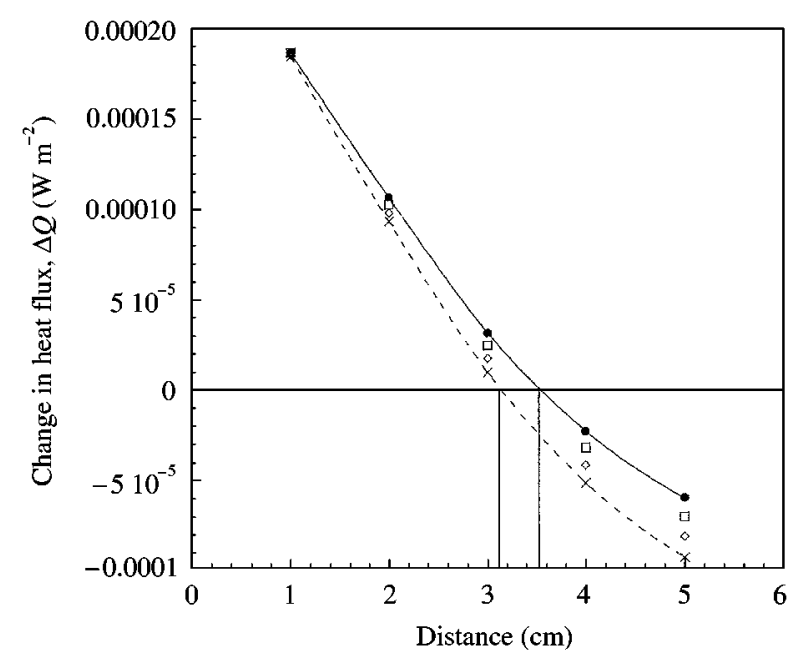

FIG. 4. Radiant flux of the background model as a function of emitter to detector distance. Compares a range of snake/soil temperature values $(290,295,300,305 \mathrm{~K})$. This shows results if areas 1 and 4 of Fig. 1 are treated as soil. $290 \mathrm{~K}, \longrightarrow-; 295 \mathrm{~K}, \square ; 300 \mathrm{~K}, \diamond ; 305 \mathrm{~K},--\times--$.

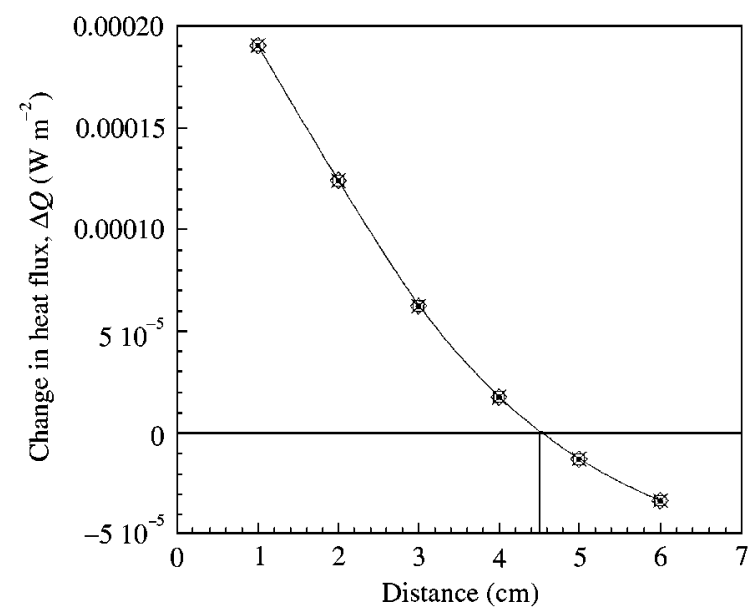

FIG. 5. Radiant flux of the background model as a function of emitter to detector distance. Compares a range of snake/soil temperature values $(290,295,300,305 \mathrm{~K})$. This shows results if areas 1 and 4 of Fig. 1 are treated as atmosphere. $290 \mathrm{~K}, \longrightarrow$ - $295 \mathrm{~K}, \square ; 300 \mathrm{~K}, \diamond ; 305 \mathrm{~K}, \times$.

question, energy conservation to a control volume, which included the pit membrane and the incident radiation, was applied. The governing equation is as follows:

$$
\dot{E}_{\text {in }}+\dot{E}_{g}-\dot{E}_{\text {out }}=\dot{E}_{\text {st }}
$$

where $\dot{E}_{i n}$ is the rate at which thermal energy enters the control volume, $\dot{E}_{\text {out }}$ the rate at which thermal energy leaves the control volume, $\dot{E}_{g}$ the rate of internal energy generation, and $\dot{E}_{s t}$ the rate of change of energy stored within the control volume.

In our case, the $\dot{E}_{\text {in }}-\dot{E}_{\text {out }}$ term was combined to form a net amount of energy in the system. This came from the configuration factor model taking into account all radiant surfaces. The model also assumed that since the pit membrane is thin, there was no internal energy generation. Equation (7) now takes the form

$$
\begin{gathered}
\dot{E}_{\text {in }}-\dot{E}_{\text {out }}=\dot{E}_{s t}, \\
Q=\rho V c_{p} \frac{\mathrm{d} T}{\mathrm{~d} t},
\end{gathered}
$$

where $Q$ is the heat flux, $\rho$ the density of the pit membrane $\left(1 \times 10^{6} \mathrm{~g} \mathrm{~m}^{-3}\right), V$ the volume of the pit membrane $\left(4.71 \times 10^{-11} \mathrm{~m}^{3}\right), c_{p}$ the specific heat of the pit membrane $\left(2.2 \mathrm{~J}^{-1} \mathrm{~g} \mathrm{~K}^{-1}\right.$, for tissue), and $\mathrm{d} T / \mathrm{d} t$ the time rate of change of temperature of the pit membrane.

Rearranging eqn (9) gives the initial heating rate of the pit membrane,

$$
\frac{\mathrm{d} T}{\mathrm{~d} t}=\frac{Q}{\rho V c_{p}} .
$$

Next, the heat flux values $(Q)$ from the simplified configuration factor model of just the emitter and the detector (areas 2 and 3 of the model) were placed into eqn (10). Figure 6 shows the initial heating rate in terms of emitter to detector distance. These values were compared to the configuration factor model containing the background. From Fig. 6, it is shown that the background had a significant effect on the detector performance. Without the background in the model, the detector to emitter distance was as far as $\sim 20 \mathrm{~cm}$ before falling below the threshold value of $0.05^{\circ} \mathrm{C} \mathrm{sec}^{-1}$. With the background included, the detector to emitter distance fell below the threshold value of $0.05^{\circ} \mathrm{C} \mathrm{sec}^{-1}$ at a distance of $3.2 \mathrm{~cm}$. Other radii of the cylinder and cone geometry were examined in order to see if the threshold would change significantly. When a radius of $10 \mathrm{~cm}$ was used instead of $100 \mathrm{~cm}$ in areas 1 and 4 for the cylinder, the threshold distance increased to $3.3 \mathrm{~cm}$, and when a radius 


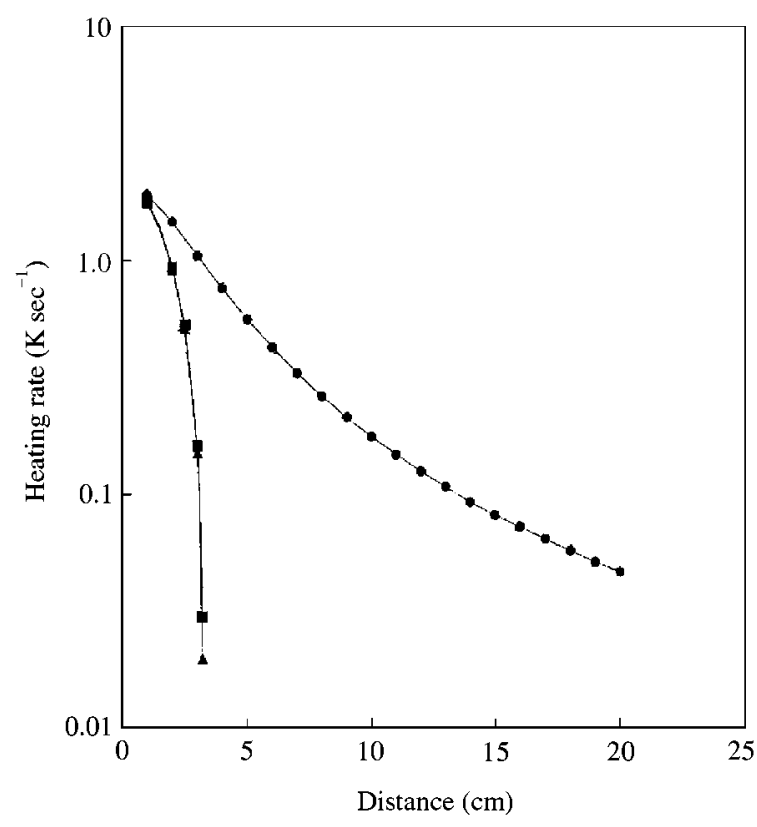

FIG. 6. Initial heating rate for the pit membrane as a function of sensor to detector distance. Three curves: two showing emitter to detector with and without the background present in the model, and one showing a conical geometry with background present. No background, - - ; background, cylinder geometry, - - ; background, cone geometry,

of $5 \mathrm{~cm}$ was used, the threshold distance increased to $3.9 \mathrm{~cm}$. Similar results were seen in the cone model when varying the radius of the base in the same manner. There was only a slight change in the emitter to detector threshold distance when comparing the different radii. Only the areas behind the prey and snake were affected by this change. These two areas have little to do with the overall emitted radiation in the model. In fact, the configuration factors for these two areas were negligible as compared to the ground and atmosphere (areas 5 and 6). As a result, the ground and atmosphere are the major contributors to the overall radiation exchange in the model.

\section{Discussion}

Previous attempts to model crotaline infrared reception or thermal sensitivity (Terashima et al., 1968; de Cock Buning, 1983b) have used relatively simple linear relationships to describe heat transfer from one object to another. Missing from this analysis was the effect background has on

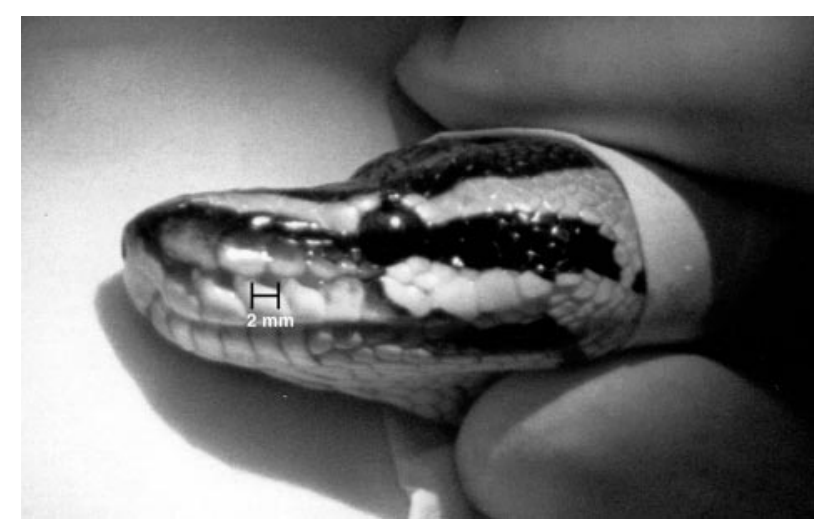

FIG. 7. Photo of Python regius (ball python) head showing four prominent pit organs on the upper jaw, each organ approximately $2 \mathrm{~mm}$ in diameter.

this process. To make an analogy with how our eyes perceive visible light, the glare of an external background source (e.g. the sun) can make an object difficult to perceive. For example, most people have had problems visualizing oncoming traffic when the glare from the windshield is too great. We have applied this same consideration of reflected energy to our infrared model. The analysis performed in 1968 by Terashima et al. used a pit organ area of $1 \mathrm{~cm}^{2}$ and a temperature differential of $16^{\circ} \mathrm{C}$. We preferred to use values closer to the Bullock and Diecke (1956) article of $3.14 \mathrm{~mm}^{2}$ for the pit organ membrane area and a $10^{\circ} \mathrm{C}$ temperature differential. We felt that these values, especially the pit organ membrane area, more closely represented the actual biological system. Our ball pythons (Python regius) possessed circular pit organs approximately $2 \mathrm{~mm}$ in diameter, which calculates to a pit organ area of $3.14 \mathrm{~mm}^{2}$ or $0.0314 \mathrm{~cm}^{2}$, quite different from the values of Terashima et al. Understandably, the model input for this area has a large effect on the radiation-gathering properties of the system. Multiple pit organs will obviously increase the total area, but even with eight pit organs $2 \mathrm{~mm}$ in diameter (see Fig. 7), we do not approach $1 \mathrm{~cm}^{2}$ total area. Additionally, the area of the emitter observably affects the results. For example, a hand would have substantially more emitting area than a mouse, and obviously would be sensed at a greater distance.

There will be a point when the radiation from the background, which is incident on the pit organ, is greater than the radiation emitted from 
the prey (see Fig. 3). This is when $\Delta Q$ will become negative. For prey detection, $\Delta Q$ must be greater than zero, otherwise the snake will no longer be able to differentiate the prey from the background. The exact detection range is determined by the sensitivity of the sensor organ. However, the limited range determined by distinguishing the prey from the background implies that a single sensor does not function well as a longrange prey detection system. It should be noted that snakes posses more than one sensor. In the case of crotalines, there are two infrared organs and boids possess an array of infrared pit organs on the upper and sometimes lower jaw. The advantages that result from having multiple sensors directed at the same receptive field will require further investigation.

The discrepancy between the previously published sensitivity of this system $\left(0.003^{\circ} \mathrm{C}\right)$ and our position of a limited-range detector can be reconciled if one views the snake's infrared system as a biological thermal detector and not as an infrared photon (or semiconductor-like) detector. We propose that far-infrared photons are absorbed by the outer cornified epidermal layer and the corresponding molecular vibrations dissipate this absorbed energy as heat. The terminal nerve masses respond with an increased firing rate if the stimulus is warmer than background (warm receptors). Therefore, a radiant stimulus (blackbody radiation) is converted into a thermal stimulus via outer matrix absorption and transferred through conduction to the snake's biological thermocouple (terminal nerve masses) with $0.003^{\circ} \mathrm{C}$ sensitivity.

This above-proposed mechanism of biological infrared detection and the comparison with semiconductor infrared detectors is meant to intentionally draw attention to different terms used in different fields. The biological value of $0.003^{\circ} \mathrm{C}$ should be viewed as the noise equivalent differential temperature (NEDT) used by infrared engineers. This sensitivity does not take into account stimulus distance from the sensor nor the background equivalent temperature. The snake's warm receptor is the same receptor type found in mammalian skin but 20 times closer to the surface and thousands of times more dense (Barrett et al., 1970). However, even with this altered geometry, we would argue from a model stand- point, that this thermal sensor cannot detect a $37^{\circ} \mathrm{C}$ infrared emitter at a half-meter distance, as stated in some reports (Newman \& Hartline, 1982). Instead, a source with a much higher temperature, i.e. soldering iron or glowing light bulb filament, is required for detection ranges approaching $50 \mathrm{~cm}$. Ultimately, the confines and limitations of the snake system are driven by the low level of energy emitted by a $37^{\circ} \mathrm{C}$ object. As shown in the results section, changing soil temperature (Fig. 4), changing atmosphere temperature (Fig. 5), or changing to a conical geometry (Fig. 6) had little effect on the overall sensing distance-less than $5 \mathrm{~cm}$ in all three cases.

Our results may well describe the boid system, as our theoretical results seem to match experimental results with boids quite well. For example, direct measurements made by de Cock Buning (1983b) placed boa and python sensing distances between 5 and $10 \mathrm{~cm}$ for a $10^{\circ} \mathrm{C}$ temperature differential $(\Delta T)$. Sensing distances greater than $10 \mathrm{~cm}$ for boids was accomplished using a $\Delta T$ greater than $10^{\circ} \mathrm{C}$. However, our theoretical model does not correlated well with experimental data from pit vipers like Agkistrodon. From de Cock Buning's measurements (1983b), Agkistrodon rhodostoma could sense at a $\Delta T$ of $10^{\circ} \mathrm{C}$ at over $20 \mathrm{~cm}$. With crotalids, anatomical differences raise the sensitivity of the infrared pit organ by 10-20 times. Anatomical factors such as membrane suspension (thermal isolation) and extreme vascularization (thermal dissipation) combine to form a much more sensitive detector. Indeed, these two attributes, missing from our model, may well give some pit vipers the ability to sense a weak stimulus, i.e. human hand or mouse, at over $20 \mathrm{~cm}$ distance.

Rodents have substantial temperature gradients occurring around their eyes and ears in the infrared, which may explain why some crotalines prefer to strike this region at the last moment instead of striking more posterior (De Cock Buning, 1985; Theodoratus et al., 1997). De Cock Buning (1983a) represented hunting behavior and prey acquisition as nine behavior phases and the importance of "warmth", "vision", "smell", and "touch" were defined for each phase by systematically blocking each sense during the different phases. In the case of crotalines and pythons, the visual system dominated early phases (head 
turning and approach). In the later phases of preparation and strike, thermal information became a more important part of the behavioral response and visual importance was slightly decreased.

As pointed out in de Cock Buning's (1983a) behavioral studies, prey acquisition is a complicated task that involves the fusion of multiple sensory inputs. The increased reliance upon thermal information later in the hunting cycle when distances are reduced, i.e. after approach, supports our assertion that the thermal sensory modality is operating over short distances. A bigger question exists with respect to the discrepancy between our model's results and experimental observations. While there is little debate regarding the importance of thermal information to crotalines and boids over short distances, there is still much theoretical work to be done to understand the experimental results showing this biological infrared system operating over extreme distances $(>20 \mathrm{~cm})$. The model we presented in this study strives to quantify this thermal input in the context of a complex thermal background. Our choice of representing this thermal sense as a function of detection distance rather than temperature threshold is an attempt to reconcile the interpretation differences between biologists and infrared engineers.

This work was funded by the Air Force Office of Scientific Research (AFOSR). The authors wish to thank Rajesh Naik for helpful suggestions during the preparation of this manuscript.

\section{REFERENCES}

Amemiya, F., Goris, R. C., Masuda, Y., Kishida, R., AtoBe, Y., IshiI, N. \& KusUnOKI, T. (1995). The surface architecture of snake infrared receptor organs. Biomed. Res. 16, 411-421.

Amemiya, F., Ushiki, T., Goris, R. C., Atobe, Y. \& KusUNOKI, T. (1996). Ultrastructure of the crotaline snake infrared pit receptors: SEM confirmation of TEM findings. Anatomical Record 246, 135-146.

Barret, R., Maderson, P. F. A. \& Meszler, R. M. (1970). The pit organs of snakes. In: Biology of the Reptilia, (Carl Gans, ed.) Vol. 2, Morphology B, pp. 277-300. New York: Academic Press.

Bullock, T. H. \& Diecke, F. P. J. (1956). Properties of an infra-red receptor. J. Physiol. 134, 47-87.

Campbell, A. L., Buning, T. J., Stone, M. O., Church, D. \& GRACE, M. S. (1999). Surface ultrastructure of pit organ, spectacle, and non pit organ epidermis of infrared imaging boid snakes: a scanning probe and scanning electron microscopy study. J. Struct. Biol. 126, 105-120.
De Cock Buning, T. (1983a). Thermal sensitivity as a specialization for prey capture and feeding in snakes. Am. Zool. 23, 363-375.

DE Cock Buning, T. (1983b). Thresholds of infrared sensitive tectal neurons in Python reticulatus, Boa contrictor, and Agkistrodon rhodostoma. J. Comp. Phys. A 151, 461-467.

De Cock Buning, T. (1985). Qualitative and quantitative explanation of the forms of heat sensitive organs in snakes. Acta Biotheoret. 34, 193-206.

De Cock Buning, T., Terashima, S. \& Goris, R. C. (1981). Crotaline pit organs analyzed as warm receptors. Cell. Mol. Neurobiol. 1, 69-85.

DRISCOLL, W. G. (1978). Blackbody radiation. In: Handbook of Optics, p. 13. New York: McGraw-Hill.

Hartline, P. H. (1974). Thermoreception in snakes. In: Handbook of Sensory Physiology (Fessard, A., Ed.) Vol. 3, pp. 297-312. New York: Springer Verlag.

HiSDAL, B. J. (1965). Reflectance of perfect diffuse and specular samples in (the integrating sphere. J. Opt. Soc. Am. 55, 1122-1128.

Howell, J. R. (1982). A Catalog of Radiation Configuration Factors, p. 161.

Hudson, R. D. (1969). Infrared System Engineering. New York: John Wiley \& Sons.

INCROPERA, F. P. \& DEWITT, D. P. (1996). Fundamentals of Heat and Mass Transfer, 4th Edn, pp. 648-651. New York: John Wiley \& Sons.

Noble, G. K. \& SCHMidT, A. (1937). Structure and function of the facial and labial pits of snakes. Proc. Am. Philos. Soc. 77, 263-288.

Newman, E. \& Hartline, P. (1982). The infrared vision of snakes. Sci. Am. 246, 98-107.

Salisbury, J. W., Wald, A. \& D'Aria, D. M. (1994). Thermal-infrared remote sensing and Kirchhoff's law: 1. Laboratory measurements. J. Geophys. Res. 99, 11897-11911.

Terashima, S., Goris, R. C. \& Katsuki, Y. (1968). Generator potential of crotaline snake infrared receptor. $J$. Neurophysiol. 31, 682-688.

Theodoratus, D. H., Chiszar, D. \& SMith, H. M. (1997). Rattlesnake orientation to prey as a function of thermal backgrounds and edges. Psychol. Record 47, 461-472.

TOGAWA, T. (1989). Non-contact skin emissivity: measurement from reflectance using step change in ambient radiation temperature. Clin. Phys. Physiol. Measurement 10, 39-48.

\section{APPENDIX}

Configuration factors were solved for the cylindrical geometry enclosure (see Fig. 1) by using equations found in Howell (1982, pp. 125, 162, 164, and 167). All energy fractions were solved using configuration factor algebra (i.e. summation rule and reciprocity relations).

The summation rule is stated as follows:

$$
\sum_{j=1}^{6} F_{i j}=1 \quad \text { for } i=1-6
$$


The following energy fractions were solved by the reciprocity relations:

$$
\begin{aligned}
& F_{13}=\frac{A_{3}}{A_{1}} F_{31}, \quad F_{14}=\frac{A_{4}}{A_{1}} F_{41}, \quad F_{32}=\frac{A_{2}}{A_{3}} F_{23}, \\
& F_{42}=\frac{A_{2}}{A_{4}} F_{24}, \quad F_{51}=\frac{A_{1}}{A_{5}} F_{15}, \\
& F_{52}=\frac{A_{2}}{A_{5}} F_{25}, \quad F_{53}=\frac{A_{3}}{A_{5}} F_{35}, \quad F_{54}=\frac{A_{4}}{A_{5}} F_{45}, \\
& F_{61}=\frac{A_{1}}{A_{6}} F_{16}, \quad F_{62}=\frac{A_{2}}{A_{6}} F_{26}, \\
& F_{63}=\frac{A_{3}}{A_{6}} F_{36}, \quad F_{64}=\frac{A_{4}}{A_{6}} F_{46}, \\
& F_{65}=\frac{A_{5}}{A_{6}} F_{56} .
\end{aligned}
$$

Since energy cannot be exchanged with itself for flat surfaces located within the same plane, the following configuration factors are zero:

$$
F_{11}=F_{12}=F_{21}=F_{22}=F_{33}=F_{34}=F_{43}=F_{44}=0 \text {. }
$$

For the cases that involved the incident radiation from area 5 or 6 onto the rest of the geometry, the configuration factors listed in Howell (1982) were divided by two. This was due to our model having the cylinder divided into two sections, whereas Howell's (1982) geometry had the cylinder modeled as a whole. Cases where there was radiation exchange between areas 5 and 6 , the configuration factors were solved analytically from equations in Howell (1982, p. 167).

Configuration factors between two finite areas,

$$
\begin{aligned}
F_{15}= & \frac{1}{4}\left\{1+\frac{1}{\left(R^{2}-1\right)}\left[H\left(4 R^{2}+H^{2}\right)^{1 / 2}\right.\right. \\
& \left.\left.-\left(\left(1+R^{2}+H^{2}\right)^{2}-4 R^{2}\right)^{1 / 2}\right]\right\},
\end{aligned}
$$

where

$$
\begin{array}{r}
R=\frac{r_{5}}{r_{2}} \quad \text { and } H=\frac{a}{r_{2}} \quad \text { (Howell, 1982, p. 164), } \\
F_{23}=\frac{1}{2}\left\{X-\left[X^{2}-4\left(\frac{R_{3}}{R_{2}}\right)^{2}\right]^{1 / 2}\right\}, \quad \text { (A.5) }
\end{array}
$$

where

$$
R_{2}=\frac{r_{2}}{a}, \quad R_{3}=\frac{r_{3}}{a} \quad \text { and } \quad X=1+\frac{1+R_{3}^{2}}{R_{2}^{2}}
$$

(Howell, 1982, p. 125),

$$
\begin{aligned}
F_{25}= & \frac{1}{4}\left\{1-R^{2}-H^{2}\right. \\
& \left.+\left[\left(1+R^{2}+H^{2}\right)^{2}-4 R^{2}\right]^{1 / 2}\right\},
\end{aligned}
$$

where

$$
\begin{aligned}
R=\frac{r_{5}}{r_{2}} \quad \text { and } \quad H=\frac{a}{r_{2}} \quad \text { (Howell, 1982, p. 162), } \\
F_{26}=\frac{1}{4}\left\{1-R^{2}-H^{2}\right. \\
\left.\quad+\left[\left(1+R^{2}+H^{2}\right)^{2}-4 R^{2}\right]^{1 / 2}\right\}, \quad \text { (A.7 }
\end{aligned}
$$

where

$$
R=\frac{r_{6}}{r_{2}} \quad \text { and } \quad H=\frac{a}{r_{2}} \quad \text { (Howell, 1982, p. 162), }
$$

$$
\begin{aligned}
F_{35}= & \frac{1}{4}\left\{1-R^{2}-H^{2}\right. \\
& \left.+\left[\left(1+R^{2}+H^{2}\right)^{2}-4 R^{2}\right]^{1 / 2}\right\},
\end{aligned}
$$

where

$R=\frac{r_{5}}{r_{3}} \quad$ and $\quad H=\frac{a}{r_{3}} \quad$ (Howell, 1982, p. 162),

where

$$
\begin{aligned}
F_{36}= & \frac{1}{4}\left\{1-R^{2}-H^{2}\right. \\
& \left.+\left[\left(1+R^{2}+H^{2}\right)^{2}-4 R^{2}\right]^{1 / 2}\right\},
\end{aligned}
$$

$R=\frac{r_{6}}{r_{3}} \quad$ and $\quad H=\frac{a}{r_{3}} \quad$ (Howell, 1982, p. 162),

$$
\begin{aligned}
F_{45}= & \frac{1}{4}\left\{1+\frac{1}{\left(R^{2}-1\right)}\left[H\left(4 R^{2}+H^{2}\right)^{1 / 2}\right.\right. \\
& \left.\left.-\left(\left(1+R^{2}+H^{2}\right)^{2}-4 R^{2}\right)^{1 / 2}\right]\right\},
\end{aligned}
$$


where

$R=\frac{r_{5}}{r_{3}} \quad$ and $\quad H=\frac{a}{r_{3}} \quad$ (Howell, 1982, p. 164),

$$
F_{46}=\frac{1}{4}\left\{1+\frac{1}{\left(R^{2}-1\right)}\left[H\left(4 R^{2}+H^{2}\right)^{1 / 2}+4 \tan ^{-1}\left(\frac{1}{2 R}\right)\right]\right.
$$$$
\left.\left.-\left(\left(1+R^{2}+H^{2}\right)^{2}-4 R^{2}\right)^{1 / 2}\right]\right\},
$$

where

$R=\frac{r_{6}}{r_{3}} \quad$ and $\quad H=\frac{a}{r_{3}} \quad$ (Howell, 1982, p. 164), $\quad R=\frac{r_{5}}{a}, \quad F_{56}=F_{65} \quad$ (Howell, 1982, p. 167).

$$
F_{56}=\frac{1}{\pi^{2}}\left[4 R \ln (2 R)+\frac{1-4 R^{2}}{2 R} \ln \left(4 R^{2}+1\right)\right.
$$

where

$$
R=\frac{r_{5}}{a}, \quad F_{56}=F_{65} \quad \text { (Howell, 1982, p. 167). }
$$

